J. Korean Math. Soc. 50 (2013), No. 1, pp. 173-187

http://dx.doi.org/10.4134/JKMS.2013.50.1.173

\title{
RICCATI EQUATION IN QUADRATIC OPTIMAL CONTROL PROBLEM OF DAMPED SECOND ORDER SYSTEM
}

\author{
Junhong Ha AND ShIN-ICHI NAKAGIRI
}

\begin{abstract}
This paper studies the properties of solutions of the Riccati equation arising from the quadratic optimal control problem of the general damped second order system. Using the semigroup theory, we establish the weak differential characterization of the Riccati equation for a general class of the second order distributed systems with arbitrary damping terms.
\end{abstract}

\section{Introduction}

It is well understood that the feedback control law of the linear quadratic optimal control problem can be determined by the solution of a proper Riccati equation. The studies of Riccati equations for parabolic and hyperbolic control systems has been developed in full extent (cf. [5], [8], [19], [20]). In this paper we consider the second order damped evolution equation system described by

$$
\begin{cases}\frac{d^{2} y}{d t^{2}}+A_{2} \frac{d y}{d t}+A_{1} y=f+B u & \text { in }(0, T), \\ z=C y & \text { on }[0, T],\end{cases}
$$

where $A_{1}$ and $A_{2}$ are operators defined on Hilbert spaces, and $B$ and $C$ are control and observation operators. When $A_{2} \equiv 0$, Lions [20] established the optimal control problem for (1.1) with related Riccati equations based on the Galerkin finite approximation method which has many restrictions and formal calculations. As a way to avoid these formal calculations, the quadratic optimal control theory has been formulated in the semigroup theoretical framework for problems having unbounded controls and observations. For the case of analytic semigroup with unbounded $B$ and $C$ we refer to Bucci [6], Flandoli [11], Lasiecka and Triggiani [18, 19], Triggiani [25], and for the case of strongly continuous semigroup under stronger assumptions on $B$ and $C$ to Pritchard and Salamon [23]. For the specific types of hyperbolic equations with damping terms, for example, $A_{2}=A_{1}^{\frac{1}{2}}$ or $A_{2}=A_{1}$ or $A_{2}$ is a constant operator,

Received February 17, 2012; Revised May 11, 2012.

2010 Mathematics Subject Classification. Primary 49J20, 49J27.

Key words and phrases. Riccati equation, quadratic optimal control problem, hyperbolic equation with damping term. 
many researchers have dealt with the quadratic optimal control problems. In particular, Triggiani [25] has treated the wave-like and plate-like systems and solved the Riccati equations throughout observing the states on some larger observation space. However, we can not find any result of driving the Riccati equation which appears in solving the quadratic optimal control problem for (1.1).

The purpose of this paper is to solve the quadratic optimal control problem based on the semigroup theory. That is, we rewrite the state of (1.1) as the first order evolution equation and derive the Riccati equation. Since the system (1.1) will be defined on any Gelfand fivefold, we will be faced with difficulty in order to treat more general unboundness occurs in setting the control function $u$ and the observation operator $B$. Since the differential operators $A_{1}, A_{2}$ are chosen to be arbitrary, we can not expect that the differential operator of the first order system transformed by (1.1) generates an analytic semigroup or also is self-adjoint. This indicates that we can not deal with the Riccati equation on the spaces of fractional powers as in [23], [25]. Further the assumptions used in [23] may not be checked directly in our setting. Hence we shall solve the Riccati equation using the ideas due to Haraux on extrapolation space technique. For the technique we can refer to Haraux [15], Banks, Kappel and Wang [4] and Tanabe [24]. Thus we shall study the Riccati equation using abstract approach developed in Ahmed and Teo [1] based on the works [3, 24].

In Section 2, we review the problem of the generation of a contraction semigroup on the extrapolation space (cf. [4], [24]), and we show the equivalence between the mild solution defined by the semigroup and the weak solution. Also we study the decoupling problem for the system with an unbounded control and observation.

In Section 3, we solve the problem of the existence and uniqueness of solutions for the Riccati equation by the iteration method. Finally we write down the Riccati equation into the componentwise equations.

\section{Notations and semigroup formulations}

Let $V_{i}, i=1,2$ be real separable Hilbert spaces with inner products $(\cdot, \cdot)_{V_{i}}$ and norms $\|\cdot\|_{V_{i}}$ and $V_{i}^{\prime}$ be the dual space of $V_{i}$. The similar notations on Hilbert spaces will be used in this paper. Let $H$ be a real pivot Hilbert space with inner product $(\cdot, \cdot)_{H}$ and norm $|\cdot|$. Assume that each pair $\left(V_{i}, H\right)$ is a Gelfand triple space with the notation, $V_{i} \hookrightarrow H \equiv H^{\prime} \hookrightarrow V_{i}^{\prime}$. Let $T>0$ be fixed.

Let $a_{i}(\phi, \varphi)$ be a symmetric bilinear form defined on $V_{i}$ satisfying

(2.1) there exists $c_{i}>0$ such that $\left|a_{i}(\phi, \varphi)\right| \leq c_{i}\|\phi\|_{V_{i}}\|\varphi\|_{V_{i}}$ for all $\phi, \psi \in V_{i}$

and there exist $\alpha_{i}>0, \lambda_{i}>0$ such that

$$
a_{i}(\phi, \phi)+\lambda_{i}|\phi|^{2} \geq \alpha_{i}\|\phi\|_{V_{i}}^{2} \text { for all } \phi \in V_{i} .
$$


In (2.2) $\lambda_{1}=0$ is supposed. Condition (2.1) defines the operator $A_{i} \in \mathcal{L}\left(V_{i}, V_{i}^{\prime}\right)$ satisfying $a_{i}(\phi, \varphi)=\left\langle A_{i} \phi, \varphi\right\rangle_{V_{i}^{\prime}, V_{i}}$ for all $\phi, \varphi \in V_{i}$. Also condition (2.2) gives an equivalent norm on $V_{1}$ using $(\phi, \varphi)_{V_{1}}=a_{1}(\phi, \varphi)$ and this norm induced by $a_{1}$ is used in this paper.

We suppose that $V_{1}$ is continuously embedded in $V_{2}$. Then we see that $V_{1} \hookrightarrow V_{2} \hookrightarrow H \equiv H^{\prime} \hookrightarrow V_{2}^{\prime} \hookrightarrow V_{1}^{\prime}$ and the equalities $\langle\phi, \varphi\rangle_{V^{\prime}, V}=\langle\phi, \varphi\rangle_{V_{2}^{\prime}, V_{2}}$ for $\phi \in V_{2}^{\prime}, \varphi \in V$ and $\langle\phi, \varphi\rangle_{V_{1}^{\prime}, V_{1}}=(\phi, \varphi)_{H}$ for $\phi \in H, \varphi \in V$ hold.

Using the operators $A_{i}, i=1,2$ one can consider the damped second order evolution equation:

$$
\left\{\begin{array}{l}
\frac{d^{2} y}{d t^{2}}+A_{2} \frac{d y}{d t}+A_{1} y=f \text { in }(0, T), \\
y(0)=y_{0} \in V, \quad \frac{d y}{d t}(0)=y_{1} \in H .
\end{array}\right.
$$

In [13], it is proved that (2.3) has a unique weak solution $y$ satisfying $y \in C([0, T] ; V) \cap C^{1}([0, T] ; H) \cap W(0, T), W(0, T)=\left\{g \in L^{2}(0, T ; V) \mid \frac{d g}{d t} \in\right.$ $\left.L^{2}\left(0, T ; V_{2}\right), \frac{d^{2} g}{d t^{2}} \in L^{2}\left(0, T ; V^{\prime}\right)\right\}$, and $y$ satisfies

$$
\left\{\begin{array}{l}
\frac{d^{2}}{d t^{2}}\langle y(\cdot), \phi\rangle_{V^{\prime}, V}+\frac{d}{d t} a_{2}(y(\cdot), \phi)+a_{1}(y(\cdot), \phi)=\langle f(\cdot), \phi\rangle_{V_{2}^{\prime}, V_{2}}, \quad \forall \phi \in V, \\
y(0)=y_{0} \in V, \quad \frac{d y}{d t}(0)=y_{1} \in H,
\end{array}\right.
$$

where $f \in L^{2}\left(0, T ; V_{2}^{\prime}\right)$.

In order to reduce the equation (2.3) to be the first order evolution equation, let us introduce product Hilbert spaces $\mathcal{V}_{1}=V_{1} \times V_{1}, \mathcal{V}_{2}=V_{1} \times V_{2}$ and $\mathcal{H}=V_{1} \times H$, and we define a bilinear form $a$ on $\mathcal{V}_{1}$ as $a(\Phi, \Psi)=-\left(\phi_{2}, \psi_{1}\right)_{V}+$ $a_{1}\left(\phi_{1}, \psi_{2}\right)+a_{2}\left(\phi_{2}, \psi_{2}\right)$. Since $V_{1} \hookrightarrow V_{2}, a$ is clearly a bounded linear functional on $\mathcal{V}$. Since $V_{1} \hookrightarrow V_{2} \hookrightarrow H \hookrightarrow V_{2}^{\prime} \hookrightarrow V_{1}^{\prime}$, it is easily verified that $\mathcal{V}_{1} \hookrightarrow \mathcal{H}_{2} \hookrightarrow$ $\mathcal{H} \hookrightarrow \mathcal{V}_{2}^{\prime} \hookrightarrow \mathcal{V}_{1}^{\prime}$.

Then the equation (2.4) can be rewritten by

$$
\left\{\begin{array}{l}
\left\langle\mathbf{y}^{\prime}, \Phi\right\rangle_{\mathcal{V}^{\prime}, \mathcal{V}}+a(\mathbf{y}, \Phi)=\langle\mathbf{f}, \Phi\rangle_{\mathcal{V}_{1}^{\prime}, \mathcal{V}_{1}}, \Phi \in \mathcal{V}_{1}, \\
\mathbf{y}(0)=\mathbf{y}_{0}=\left(y_{0}, y_{1}\right) \in \mathcal{H},
\end{array}\right.
$$

where $\mathbf{f}=(0, f)$.

The definition of $a$ implies that there exists $\tilde{\mathcal{A}} \in \mathcal{L}\left(\mathcal{V}_{1}, \mathcal{V}_{1}^{\prime}\right)$ such that $a(\Phi, \Psi)$ $=\langle\tilde{\mathcal{A}} \Phi, \Psi\rangle_{\mathcal{V}_{1}^{\prime}, \mathcal{V}_{1}}$. Putting $\mathbf{y}=\left(y, y^{\prime}\right) \in L^{2}\left(0, T ; \mathcal{V}_{2}\right) \subset L^{2}(0, T ; \mathcal{H})$, one get the first order evolution equation given by

$$
\left\{\begin{array}{c}
\frac{d}{d t} \mathbf{y}=\mathcal{A} \mathbf{y}+\mathbf{f} \text { in }(0, T), \\
\mathbf{y}(0)=\mathbf{y}_{0}=\left(y_{0}, y_{1}\right) \in \mathcal{H},
\end{array}\right.
$$

where $\mathcal{D}(\mathcal{A})=\left\{\Phi=(\phi, \psi) \in \mathcal{H} \mid \psi \in V_{1}\right.$ and $\left.A_{1} \phi+A_{2} \psi \in H\right\}$ and $\mathcal{A} \Phi=$ $\left(\psi,-A_{1} \phi-A_{2} \psi\right)$. Note that $-\mathcal{A}$ is the restriction to $\mathcal{D}(\mathcal{A})$ of the operator $\tilde{\mathcal{A}}$ and the operator $\mathcal{A}$ generates a $C_{0}$-contraction semigroup $S(t)$ on $\mathcal{H}$, that is, 
$|S(t)|_{\mathcal{H}} \leq e^{\lambda t}$ for any $\lambda \geq \lambda_{2}$ (See [3]). Hence we can define the mild solution $\mathbf{y} \equiv \mathbf{y}\left(t ; \mathbf{y}_{0}, \mathbf{f}\right)$ for $(2.5)$ by

$$
\mathbf{y}(t)=S(t) \mathbf{y}_{0}+\int_{0}^{t} S(t-s) \mathbf{f}(s) d s,
$$

where $\mathbf{y}_{0} \in \mathcal{H}, \mathbf{f}=(0, f), f \in L^{2}(0, T ; H)$. Since this expression (2.6) is not enough to treat $f \in L^{2}\left(0, T ; V_{2}^{\prime}\right)$, a generalized formula is required and it can be accomplished using the adjoint operator $\mathcal{A}^{*}$, which is given by $\mathcal{A}^{*} \Phi=$ $\left(-\psi, A_{1} \phi-A_{2} \psi\right)$ with $\mathcal{D}\left(\mathcal{A}^{*}\right)=\left\{\Phi=(\phi, \psi) \in \mathcal{H} \mid \psi \in V\right.$ and $\left.A_{1} \phi-A_{2} \psi \in H\right\}$. Since the operator $\lambda I-\mathcal{A}^{*}$ is invertible in $\mathcal{H}$ for all $\lambda>\lambda_{2}>0$, we can define an inner product on $\mathcal{Y}=\mathcal{D}\left(\mathcal{A}^{*}\right)$ by $(\Phi, \Psi)_{\mathcal{Y}}=\left(\left(\lambda-\mathcal{A}^{*}\right) \Phi,\left(\lambda-\mathcal{A}^{*}\right) \Psi\right)_{\mathcal{H}}$ and the norm by $\|\Phi\|_{\mathcal{Y}}^{2}=(\Phi, \Phi) \mathcal{Y}$. Since this norm is equivalent to the graph norm of $\mathcal{A}^{*}$, the adjoint semigroup $S^{*}(t)$ is also a $C_{0}$-semigroup on $\mathcal{Y}$ whose the infinitesimal generator is $\mathcal{A}^{*}$ (see $[22$, p. 39]). Also we can build up a Gelfand triple form $\mathcal{Y} \hookrightarrow \mathcal{H} \hookrightarrow \mathcal{Y}^{\prime}$. Note that we do not have any information on $\mathcal{Y} \hookrightarrow \mathcal{V}$, but on $\mathcal{Y}^{\prime} \hookrightarrow \mathcal{V}^{\prime}$.

Now let us define a bilinear form $\hat{a}$ on $\mathcal{H} \times \mathcal{Y}$ by $\hat{a}(\Phi, \Psi)=\left(\Phi, \mathcal{A}^{*} \Psi\right)_{\mathcal{H}}$. Since $\mathcal{A}^{*}$ is bounded on $\mathcal{Y}$, there exists a $\hat{\mathcal{A}} \in \mathcal{L}\left(\mathcal{H}, \mathcal{Y}^{\prime}\right)$ such that $\langle\hat{\mathcal{A}} \Phi, \Psi\rangle_{\mathcal{Y}^{\prime}, \mathcal{Y}}=$ $\left(\Phi, \mathcal{A}^{*} \Psi\right)_{\mathcal{H}}$ for $\Phi \in \mathcal{H}, \Psi \in \mathcal{Y}$. Note that $\hat{\mathcal{A}}^{*}=\mathcal{A}^{*}$ and $\mathcal{A}^{* *}=\hat{\mathcal{A}}$.

The following theorems and lemmas are crucial for solving our problem and their proofs are given in [3].

Theorem 2.1. The operator $\hat{\mathcal{A}}$ generates a $C_{0}$-semigroup $\hat{S}(t)$ on $\mathcal{Y}^{\prime}$. Furthermore, the operator $\hat{\mathcal{A}}$ is an extension of $\mathcal{A}$ from $\mathcal{D}(\mathcal{A})$ to $\mathcal{H}$ and $\hat{S}(t)$ is an extension of $S(t)$ from $\mathcal{H}$ to $\mathcal{Y}^{\prime}$.

According to the semigroup properties of $\hat{S}(t)$, we can define the function $\hat{\mathbf{y}}_{m} \equiv \hat{\mathbf{y}}\left(t ; \mathbf{y}_{0}, \mathbf{f}\right)$ belonging to $C\left([0, T] ; \mathcal{Y}^{\prime}\right)$ given by

$$
\hat{\mathbf{y}}(t)=\hat{S}(t) \mathbf{y}_{0}+\int_{0}^{t} \hat{S}(t-s) \mathbf{f}(s) d s
$$

as the mild solution of the initial value problem

$$
\left\{\begin{array}{l}
\frac{d}{d t} \hat{\mathbf{y}}=\hat{\mathcal{A}} \hat{\mathbf{y}}+\mathbf{f} \quad \text { in }(0, T), \\
\hat{\mathbf{y}}(0)=\mathbf{y}_{0} \in \mathcal{Y}^{\prime}
\end{array}\right.
$$

where $\mathbf{f} \in L^{2}\left(0, T ; \mathcal{Y}^{\prime}\right)$.

Lemma 2.2. Let $\mathbf{y}_{0} \in \mathcal{Y}^{\prime}, \mathbf{f} \in L^{2}\left(0, T ; \mathcal{Y}^{\prime}\right)$ and $\hat{\mathbf{y}}(t)$ be the mild solution $(2.7)$. Then for every $\xi \in \mathcal{Y}$, the function $\langle\hat{\mathbf{y}}(t), \xi\rangle_{\mathcal{Y}^{\prime}, \mathcal{Y}}$ is absolutely continuous on $[0, T]$ and satisfies

$$
\frac{d}{d t}\langle\hat{\mathbf{y}}(t), \xi\rangle \mathcal{Y}^{\prime}, \mathcal{Y}=\left\langle\hat{\mathbf{y}}(t), \mathcal{A}^{*} \xi\right\rangle_{\mathcal{Y}^{\prime}, \mathcal{Y}}+\langle\mathbf{f}(t), \xi\rangle_{\mathcal{Y}^{\prime}, \mathcal{Y}}
$$

for almost everywhere in $(0, T)$. 

by

The function $\mathbf{p} \in C([0, T] ; \mathcal{Y})$, which will be used in the next section, defined

$$
\mathbf{p}(t)=S^{*}(T-t) \mathbf{p}_{f}+\int_{t}^{T} S^{*}(s-t) \mathbf{g}(s) d s,
$$

is the mild solution of the terminal value problem

$$
\left\{\begin{array}{l}
-\frac{d}{d t} \mathbf{p}=\mathcal{A}^{*} \mathbf{p}+\mathbf{g} \text { in }(0, T) \\
\mathbf{p}(T)=\mathbf{p}_{f} \in \mathcal{Y}
\end{array}\right.
$$

where $\mathbf{g} \in L^{2}(0, T ; \mathcal{Y})$.

Lemma 2.3. Assume $\mathbf{p}_{f} \in \mathcal{Y}, \mathbf{g} \in L^{2}(0, T ; \mathcal{Y})$ and $\mathbf{p}(t)$ is the mild solution (2.8). Then for every $\xi \in \mathcal{H}$, the function $(\mathbf{p}(t), \xi)_{\mathcal{H}}$ is absolutely continuous on $[0, T]$ and satisfies

$$
-\frac{d}{d t}(\mathbf{p}(t), \xi)_{\mathcal{H}}=\langle\mathbf{p}(t), \hat{\mathcal{A}} \xi\rangle_{\mathcal{Y}, \mathcal{Y}^{\prime}}+(\mathbf{g}(t), \xi)_{\mathcal{H}}
$$

for almost all $t \in(0, T)$.

Theorem 2.4. For $\mathbf{y}_{0} \in \mathcal{H}, f \in L^{2}(0, T ; H)$, the weak solution $\mathbf{y}_{w}$ of $(2.5)$ is equal to the mild solution $\mathbf{y}_{m}$ of (2.6). For $\mathbf{y}_{0} \in \mathcal{H}, f \in L^{2}\left(0, T ; V_{2}^{\prime}\right)$, we have $\mathbf{y}_{w}=\hat{\mathbf{y}}_{m}$.

Remark 2.5. If $\mathbf{y}_{0} \in \mathcal{H}$, then $\hat{\mathbf{y}}_{m}(v ; t) \in \mathcal{H}$ for each $t \in[0, T]$. Since $y \in$ $C([0, T] ; V)$ and $y^{\prime} \in C([0, T] ; H)$, we have $\hat{\mathbf{y}}_{m}=\mathbf{y}_{w}=\mathbf{y} \in C([0, T] ; \mathcal{H})$.

\section{A Riccati equation for an observation on $\mathcal{Y}^{\prime}$}

In this section we introduce formally the Riccati equation and prove that it has a solution using the semigroup theory. First let us introduce some spaces and operators. Let $U$ and $M$ be Hilbert spaces of controls and observation variables, respectively. For the observation and controller we assume that $\mathcal{C} \in$ $L^{\infty}\left(0, T ; \mathcal{L}\left(\mathcal{Y}^{\prime}, M\right)\right)$ and $\mathcal{B} \in L^{\infty}\left(0, T ; \mathcal{L}\left(U, \mathcal{Y}^{\prime}\right)\right)$. Let the regulator operator $R$ satisfy $R \in L^{\infty}(0, T ; \mathcal{L}(U))$ and

$(R v(t), v)_{U}=(v, R v(t))_{U} \geq \gamma\|v\|_{U}^{2}, \forall v \in U$ for some $\gamma>0$ and a.e. $t \in[0, T]$.

Let $\Lambda_{M}$ (resp. $\Lambda_{U}$ ) be the canonical isomorphism from $M$ to $M^{\prime}$ (resp. from $U$ to $\left.U^{\prime}\right)$. Consider a parabolic control system in $\mathcal{Y}^{\prime}$ :

$$
\left\{\begin{array}{l}
\frac{d}{d t} \mathbf{y}(v)=\hat{\mathcal{A}} \mathbf{y}(v)+\mathcal{B} v+\mathbf{f} \text { in }(0, T), \\
\mathbf{y}(v ; 0)=\mathbf{y}_{0}=\left(y_{0}, y_{1}\right) \in \mathcal{Y}^{\prime}
\end{array}\right.
$$

where $\mathbf{f} \in L^{2}\left(0, T ; \mathcal{Y}^{\prime}\right)$. Consider a quadratic cost functional on $U$ defined by

$$
J(v)=\int_{0}^{T}\left\|\mathcal{C} \mathbf{y}(v ; t)-\mathbf{z}_{d}(t)\right\|_{M}^{2} d t+\int_{0}^{T}(R v(t), v)_{U} d t
$$


where $\mathbf{z}_{d} \in L^{2}(0, T ; M)$. By assumptions above and Lemma 2.2 we know that for each $\xi \in \mathcal{D}\left(\mathcal{A}^{*}\right)$ the solution $\mathbf{y} \in C\left([0, T] ; \mathcal{Y}^{\prime}\right)$ of $(3.1)$ satisfies

$$
\frac{d}{d t}\langle\mathbf{y}(t), \xi\rangle_{\mathcal{Y}^{\prime}, \mathcal{Y}}=\left\langle\mathbf{y}(t), \mathcal{A}^{*} \xi\right\rangle_{\mathcal{Y}^{\prime}, \mathcal{Y}}+\langle\mathcal{B} v(t)+\mathbf{f}(t), \xi\rangle_{\mathcal{Y}^{\prime}, \mathcal{Y}} \quad \text { a.e. } \quad t \in(0, T)
$$

and there is a unique optimal control $u$ such that

$$
J(u)=\inf _{v \in U} J(v)
$$

because the map $v \rightarrow \mathbf{y}(v)$ is affine. Since the map is Fréchet differentiable (see, [14]), the optimal control $u$ is also characterized by

$$
\begin{aligned}
& \int_{0}^{T}\left\langle\mathcal{C}^{*} \Lambda_{M}\left(\mathcal{C} \mathbf{y}(u)-\mathbf{z}_{d}\right)(t), \mathbf{y}(v ; t)-\mathbf{y}(u ; t)\right\rangle_{\mathcal{Y}, \mathcal{Y}^{\prime}} d t \\
& +\int_{0}^{T}(R u(t), v-u)_{U} d t \geq 0, \forall v \in U,
\end{aligned}
$$

which is equivalent to

$$
\left(\Lambda_{U}^{-1} \mathcal{B}^{*} \mathbf{p}(u)+R u, v-u\right)_{U} \geq 0 \text { for all } v \in U,
$$

where $\mathbf{p} \in C([0, T] ; \mathcal{Y})$ is the mild solution of the adjoint state equation:

$$
\left\{\begin{array}{l}
-\frac{d}{d t} \mathbf{p}(u)=\mathcal{A}^{*} \mathbf{p}(u)+\mathcal{C}^{*} \Lambda_{M}\left(\mathcal{C} \mathbf{y}(u)-\mathbf{z}_{d}\right) \text { in }(0, T), \\
\mathbf{p}(T ; 0)=\mathbf{0}
\end{array}\right.
$$

Note that $\mathbf{p}$ of (3.4) satisfies (2.9) with $\mathbf{g}(t)=\mathcal{C}^{*} \Lambda_{M}\left(\mathcal{C} \mathbf{y}(u)-\mathbf{z}_{d}\right)(t)$. Since $U$ is a Hilbert space, the optimal control $u$ satisfies

$$
\Lambda_{U}^{-1} \mathcal{B}^{*} \mathbf{p}(u)+R u=0 .
$$

Here we remark that $R$ has a bounded inverse $R^{-1} \in \mathcal{L}\left(L^{\infty}(0, T ; U), U\right)$. Hence one get the following theorem.

Theorem 3.1. The optimal control $u=-R^{-1} \Lambda_{U}^{-1} \mathcal{B}^{*} \mathbf{p}$ is determined by the system of the following equations:

$$
\begin{aligned}
& \left\{\begin{array}{l}
\frac{d}{d t} \mathbf{y}=\hat{\mathcal{A}} \mathbf{y}-\mathcal{B} R^{-1} \Lambda_{U}^{-1} \mathcal{B}^{*} \mathbf{p}+\mathbf{f} \quad \text { in } \quad(0, T), \\
\mathbf{y}(0)=\mathbf{y}_{0} \in \mathcal{Y}^{\prime}, \quad \mathbf{y} \in C\left([0, T] ; \mathcal{Y}^{\prime}\right)
\end{array}\right. \\
& \left\{\begin{array}{l}
-\frac{d}{d t} \mathbf{p}=\mathcal{A}^{*} \mathbf{p}+\mathcal{C}^{*} \Lambda_{M}\left(\mathcal{C} \mathbf{y}-\mathbf{z}_{d}\right) \quad \text { in }(0, T), \\
\mathbf{p}(T ; 0)=\mathbf{0}, \quad \mathbf{p} \in C([0, T] ; \mathcal{Y})
\end{array}\right.
\end{aligned}
$$

Note that one has to solve the coupled system given by (3.6) and (3.7) to find p. But it is inconvenient because the time flow of two equations is reverse. 
So we want to find a relation between $\mathbf{y}$ and $\mathbf{p}$. For this relation let us consider the system given by

$$
\left\{\begin{array}{c}
\frac{d}{d t} \phi-\hat{\mathcal{A}} \phi+\mathcal{D}_{1} \psi=\mathbf{f}, \quad \text { in }(s, T), \\
-\frac{d}{d t} \psi-\mathcal{A}^{*} \psi-\mathcal{D}_{2} \phi=\mathbf{g} \text { in }(s, T), \\
\phi(s)=\mathbf{h}, \quad \psi(T)=0,
\end{array}\right.
$$

where $\mathcal{D}_{1}=\mathcal{B} R^{-1} \Lambda_{U}^{-1} \mathcal{B}^{*}, \mathcal{D}_{2}=\mathcal{C}^{*} \Lambda_{M} \mathcal{C}$ and $\mathbf{g}=-\mathcal{C}^{*} \Lambda_{M} \mathbf{z}_{d}$. It is easily proved that $(3.8)$ has a unique weak solution $(\phi, \psi) \in C\left([0, T] ; \mathcal{Y}^{\prime}\right) \times C([0, T] ; \mathcal{Y})$ when the equation $\psi(T)=0$ is replaced by $\psi(T)=s$, in $J(v)$.

Since the map $\mathbf{h}$ to $\psi$, i.e., $\mathbf{y}$ to $\mathbf{p}$ are affine, one can easily notice that there are $\mathcal{P}(t)$ and $\mathbf{r}(t)$ satisfying

$$
\mathbf{p}(t)=\mathcal{P}(t) \mathbf{y}(t)+\mathbf{r}(t), \quad \mathcal{P}(t) \in \mathcal{L}\left(\mathcal{Y}^{\prime}, \mathcal{Y}\right), \quad \mathbf{r}(t) \in \mathcal{Y},
$$

where $\mathcal{P}(t)$ and $\mathbf{r}(t)$ are given as follows:

(i) we solve

$$
\left\{\begin{array}{r}
\frac{d}{d t} \beta-\hat{\mathcal{A}} \beta+\mathcal{D}_{1} \gamma=0 \\
-\frac{d}{d t} \gamma-\mathcal{A}^{*} \gamma-\mathcal{D}_{2} \beta=0 \\
\beta(s)=\mathbf{h}, \quad \gamma(T)=0
\end{array}\right\} \quad \text { in }(s, T)
$$

and then

$$
\mathcal{P}(s) \mathbf{h}=\gamma(s)
$$

(ii) we solve

and then

$$
\left\{\begin{aligned}
\frac{d}{d t} \eta-\hat{\mathcal{A}} \eta+\mathcal{D}_{1} \xi & =\mathbf{f} \\
-\frac{d}{d t} \xi-\mathcal{A}^{*} \xi-\mathcal{D}_{2} \eta & =\mathbf{g} \\
\eta(s)=0, \quad \xi(T) & =0
\end{aligned}\right\} \text { in }(s, T)
$$

$$
\mathbf{r}(s)=\xi(s) \text {. }
$$

It can be proved via a similar method as in [20] that the operator $\mathcal{P}(t)$ satisfies the following lemma.

Lemma 3.2. (1) $\langle\mathcal{P}(t) \Phi, \Psi\rangle_{\mathcal{Y}, \mathcal{Y}^{\prime}}=\langle\Phi, \mathcal{P}(t) \Psi\rangle_{\mathcal{Y}^{\prime}, \mathcal{Y}}$ for $t \in(0, T), \Phi, \Psi \in \mathcal{Y}^{\prime}$.

(2) $\langle\mathcal{P}(t) \Phi, \Phi\rangle_{\mathcal{Y}, \mathcal{Y}^{\prime}} \geq 0$ for $t \in(0, T), \Phi \in \mathcal{Y}^{\prime}$.

(3) $\mathcal{P} \in L^{\infty}\left(0, T ; \mathcal{L}\left(\mathcal{Y}^{\prime}, \mathcal{Y}\right)\right)$.

Substituting $\mathbf{p}=\mathcal{P} \mathbf{y}+\mathbf{r}$ in (3.6), we have

$$
\frac{d}{d t} \mathbf{y}-\left(\hat{\mathcal{A}}-\mathcal{D}_{1} \mathcal{P}\right) \mathbf{y}=\mathbf{f}-\mathcal{D}_{1} \mathbf{r} \text { in }(0, T)
$$

where

$$
\mathcal{D}_{1}=\mathcal{B} R^{-1} \Lambda_{U}^{-1} \mathcal{B}^{*}
$$


Since the product $\mathcal{D}_{1} \mathcal{P}$ of operators $\mathcal{D}_{1}$ and $\mathcal{P}$ in (3.9) is an operator valued function in $t$, we shall write this by $\mathcal{D}_{1} \mathcal{P}(t)$ to indicate the dependence of $t$. Then we see $\mathcal{D}_{1} \mathcal{P} \in L^{\infty}\left(0, T ; \mathcal{L}\left(\mathcal{Y}^{\prime}\right)\right)$ by Lemma 3.1 and $\mathcal{B} \in L^{\infty}\left(0, T ; \mathcal{L}\left(U, \mathcal{Y}^{\prime}\right)\right)$. The following theorem is well-known, see [24].

Theorem 3.3. The perturbed equation defined by

$$
\left\{\begin{array}{l}
\frac{d}{d t} \mathbf{y}=\left(\hat{\mathcal{A}}-\mathcal{D}_{1} \mathcal{P}(t)\right) \mathbf{y}, \quad t \in(s, T), \\
\mathbf{y}(s)=\mathbf{h} \in \mathcal{Y}^{\prime}
\end{array}\right.
$$

has a unique strongly continuous solution $\mathbf{y}$ given by $\mathbf{y}(t)=U(t, s) \mathbf{h}$, where $U(t, s)$ is the evolution operator satisfying the following properties

$$
\left\{\begin{array}{l}
U(t, t)=I \text { for } t \in[0, T] \\
U(t, r) U(r, s)=U(t, s) \text { for } 0 \leq s<r<t \leq T \\
\frac{\partial}{\partial s} U(t, s) \Phi=-U(t, s)\left(\hat{\mathcal{A}}-\mathcal{D}_{1} \mathcal{P}(s)\right) \Phi \text { for } 0<s<t<T, \quad \Phi \in \mathcal{Y}^{\prime} .
\end{array}\right.
$$

Since $\mathcal{D}_{1} \mathcal{P} \in L^{\infty}\left(0, T ; \mathcal{L}\left(\mathcal{Y}^{\prime}\right)\right), \hat{\mathcal{A}}-\mathcal{D}_{1} \mathcal{P}(t)$ is the generator of the strongly continuous evolution operator $U(t, s)$, which satisfies (3.11). Hence, the mild solution $\mathbf{y}$ of (3.9) is given by

$$
\mathbf{y}(t)=U(t, s) \mathbf{y}(s)-\int_{s}^{t} U(t, \sigma)\left(\mathbf{f}-\mathcal{D}_{1} \mathbf{r}\right)(\sigma) d \sigma, \quad t \in(s, T) .
$$

Through formal calculations, let us find the Riccati equation. First, let us substitute the following equation (the mild solution of (3.7))

$$
\mathbf{p}(t)=\int_{t}^{T} S^{*}(s-t) \mathcal{C}^{*}(s) \Lambda_{M}\left(\mathcal{C}(s) \mathbf{y}(s)-\mathbf{z}_{d}(s)\right) d s
$$

into $R u=-\Lambda_{U}^{-1} \mathcal{B}^{*} \mathbf{p}$, and then substitute (3.12) into the resulting equation. Then we obtain

$$
\begin{aligned}
& R u(t) \\
= & -\Lambda_{U}^{-1} \mathcal{B}^{*}\left(\int_{t}^{T} S^{*}(s-t) \mathcal{C}^{*}(s) \Lambda_{M} \mathcal{C}(s) U(s, t) d s\right) \mathbf{y}(t) \\
& +\Lambda_{U}^{-1} \mathcal{B}^{*}(t) \int_{t}^{T} S^{*}(s-t) \mathcal{C}^{*}(s) \Lambda_{M} \mathcal{C}(s)\left(\int_{t}^{s} U(s, \sigma)\left(\mathbf{f}-\mathcal{D}_{1} \mathbf{r}\right)(\sigma) d \sigma+\mathbf{z}_{d}(s)\right) d s .
\end{aligned}
$$

Comparing $R u(t)=-\Lambda_{U}^{-1} \mathcal{B}^{*}(t)[\mathcal{P}(t) \mathbf{y}(t)+\mathbf{r}(t)]$ with the above equation and applying $R^{-1}$, we have

$$
\mathcal{P}(t) \mathbf{h}=\int_{t}^{T} S^{*}(s-t) \mathcal{C}^{*}(s) \Lambda_{M} \mathcal{C}(s) U(s, t) d s \mathbf{h} \text { for all } \mathbf{h} \in \mathcal{Y}^{\prime}
$$

and

$$
\mathbf{r}(t)+\int_{t}^{T} S^{*}(s-t) \mathcal{P}(s)\left(\mathbf{f}-\mathcal{D}_{1} \mathbf{r}\right)(s) d s+\int_{t}^{T} S^{*}(s-t) \mathcal{C}^{*}(s) \Lambda_{M} \mathcal{C}(s) \mathbf{z}_{d}(s) d s=0 .
$$


The following calculations are formal. Differentiating $\langle\mathcal{P}(t) \Phi, \Psi\rangle_{\mathcal{Y}, \mathcal{Y}^{\prime}}$ in $t$, we have

$$
\begin{aligned}
\frac{d}{d t}\langle\mathcal{P}(t) \Phi, \Psi\rangle_{\mathcal{Y}, \mathcal{Y}^{\prime}}= & -\left\langle\mathcal{D}_{2}(t) \Phi, \Psi\right\rangle_{\mathcal{Y}, \mathcal{Y}^{\prime}}-\int_{t}^{T}\left\langle S^{*}(s-t) \mathcal{D}_{2}(s) \Phi, \hat{\mathcal{A}} \Psi\right\rangle_{\mathcal{Y}, \mathcal{Y}^{\prime}} d t \\
& -\int_{t}^{T}\left\langle S^{*}(s-t) \mathcal{D}_{2}(s) U(s, t)\left[\hat{\mathcal{A}}-\mathcal{D}_{1} \mathcal{P}(t)\right] \Phi, \Psi\right\rangle_{\mathcal{Y}, \mathcal{Y}^{\prime}} d t \\
= & -\left\langle\mathcal{D}_{2}(t) \Phi, \Psi\right\rangle_{\mathcal{Y}, \mathcal{Y}^{\prime}}-\langle\mathcal{P}(t) \Phi, \hat{\mathcal{A}} \Psi\rangle_{\mathcal{Y}, \mathcal{Y}^{\prime}}-\langle\mathcal{P}(t) \hat{\mathcal{A}} \Phi, \Psi\rangle_{\mathcal{Y}, \mathcal{Y}^{\prime}} \\
& +\left\langle\mathcal{P} \mathcal{D}_{1} \mathcal{P}(t) \Phi, \Psi\right\rangle_{\mathcal{Y}, \mathcal{Y}^{\prime}}
\end{aligned}
$$

where $\mathcal{P} \mathcal{D}_{1} \mathcal{P}(t)=\mathcal{P}(t) \mathcal{D}_{1} \mathcal{P}(t)$. Differentiating $\langle\mathbf{r}(t), \Phi\rangle_{\mathcal{Y}, \mathcal{Y}^{\prime}}$ again in $t$, we also have

$$
\frac{d}{d t}\langle\mathbf{r}(t), \Phi\rangle_{\mathcal{Y}, \mathcal{Y}^{\prime}}=\left\langle\left[\mathcal{P}\left(\mathbf{f}-\mathcal{D}_{1} \mathbf{r}\right)+\mathcal{D}_{2} \mathbf{z}_{d}\right](t), \Phi\right\rangle_{\mathcal{Y}, \mathcal{Y}^{\prime}}+\langle\mathbf{r}(t), \hat{\mathcal{A}} \Phi\rangle_{\mathcal{Y}, \mathcal{Y}^{\prime}}
$$

Hence we can deduce formally the Riccati equation given in Theorem 3.4.

Theorem 3.4. There exists a unique operator $\mathcal{P}(t)$ and a function $\mathbf{r}(t)$ which satisfy the following differential equations

$$
\left\{\begin{array}{l}
\frac{d}{d t}\langle\mathcal{P}(t) \Phi, \Psi\rangle_{\mathcal{Y}, \mathcal{Y}^{\prime}}+\langle\hat{\mathcal{A}} \Phi, \mathcal{P}(t) \Psi\rangle_{\mathcal{Y}^{\prime}, \mathcal{Y}}+\langle\mathcal{P}(t) \Phi, \hat{\mathcal{A}} \Psi\rangle_{\mathcal{Y}, \mathcal{Y}^{\prime}} \\
-\left\langle\mathcal{P} \mathcal{D}_{1} \mathcal{P}(t) \Phi, \Psi\right\rangle_{\mathcal{Y}, \mathcal{Y}^{\prime}}+\left\langle\mathcal{D}_{2}(t) \Phi, \Psi\right\rangle_{\mathcal{Y}, \mathcal{Y}^{\prime}}=0 \text { a.e. } t \in(0, T) \\
\mathcal{P}(T)=0
\end{array}\right.
$$

and

$$
\left\{\begin{array}{l}
\frac{d}{d t}\langle\mathbf{r}(t), \Psi\rangle_{\mathcal{Y}, \mathcal{Y}^{\prime}}-\langle\mathbf{r}(t), \hat{\mathcal{A}} \Psi\rangle_{\mathcal{Y}, \mathcal{Y}^{\prime}}-\left\langle\mathcal{P}\left(\mathbf{f}-\mathcal{D}_{1} \mathbf{r}\right)(t), \Psi\right\rangle_{\mathcal{Y}, \mathcal{Y}^{\prime}} \\
-\left\langle\mathcal{D}_{2} \mathbf{z}_{d}(t), \Psi\right\rangle_{\mathcal{Y}, \mathcal{Y}^{\prime}}=0 \quad \text { a.e. } \quad t \in(0, T) \\
\mathbf{r}(T)=0
\end{array}\right.
$$

for all $\Phi \in \mathcal{Y}, \Psi \in \mathcal{Y}^{\prime}$.

Since the proof of Theorem 3.4 is too long and complicated, we give an outline of the proof. For a detailed proof we refer to Ha [12] or Chapter 3, Section 4 in Lions [20].

An outline of proof. We will prove this theorem based on the principle of linearization and successive approximations. Let $\Phi \in \mathcal{Y}$ and $\Psi \in \mathcal{Y}^{\prime}$ without indicating particularly. The Riccati equation can be written as

$$
\left\{\begin{array}{l}
\frac{d}{d t}\langle\mathcal{P}(t) \Phi, \Psi\rangle \mathcal{Y}, \mathcal{Y}^{\prime}+\left\langle\left(\hat{\mathcal{A}}-\mathcal{D}_{1} \mathcal{P}(t)\right) \Phi, \mathcal{P}(t) \Psi\right\rangle_{\mathcal{Y}^{\prime}, \mathcal{Y}} \\
+\left\langle\mathcal{P}(t) \Phi,\left(\hat{\mathcal{A}}-\mathcal{D}_{1} \mathcal{P}(t)\right) \Psi\right\rangle \mathcal{Y}, \mathcal{Y}^{\prime} \\
+\left\langle\left(\mathcal{P} \mathcal{D}_{1} \mathcal{P}(t)+\mathcal{D}_{2}(t)\right) \Phi, \Psi\right\rangle \mathcal{Y}_{\mathcal{Y}^{\prime}}=0 \text { a.e. } t \in(0, T) \\
\mathcal{P}(T)=0
\end{array}\right.
$$

We shall solve (3.13) by iteration. For $n=0$, we set

$$
\mathcal{P}_{0}(t)=\int_{t}^{T} U^{*}(\sigma, t) \mathcal{D}_{2}(\sigma) U(\sigma, t) d \sigma, \quad t \in[0, T] .
$$


Assume that $\mathcal{P}_{n-1} \in L^{\infty}\left(0, T ; \mathcal{L}\left(\mathcal{Y}^{\prime}, \mathcal{Y}\right)\right)$ is already given. Let $\mathcal{P}_{n}(t)$ be the solution of the linearized version of $(3.13)$ given by

$$
\left\{\begin{array}{l}
\frac{d}{d t}\left\langle\mathcal{P}_{n}(t) \Phi, \Psi\right\rangle_{\mathcal{Y}, \mathcal{Y}^{\prime}}+\left\langle\left(\hat{\mathcal{A}}-\mathcal{D}_{1} \mathcal{P}_{n-1}(t)\right) \Phi, \mathcal{P}_{n}(t) \Psi\right\rangle_{\mathcal{Y}^{\prime}, \mathcal{Y}} \\
+\left\langle\mathcal{P}_{n}(t) \Phi,\left(\hat{\mathcal{A}}-\mathcal{D}_{1} \mathcal{P}_{n-1}(t)\right) \Psi\right\rangle \mathcal{Y}, \mathcal{Y}^{\prime} \\
+\left\langle\left(\mathcal{P}_{n-1} \mathcal{D}_{1} \mathcal{P}_{n-1}(t)+\mathcal{D}_{2}(t)\right) \Phi, \Psi\right\rangle_{\mathcal{Y}, \mathcal{Y}^{\prime}}=0 \quad \text { a.e. } t \in(0, T), \\
\mathcal{P}_{n}(T)=0, \quad n=1,2,3, \ldots
\end{array}\right.
$$

Set $\Delta \equiv\{(t, s) \mid 0<t \leq s<T\}$. Since $\mathcal{D}_{1} \mathcal{P}_{n-1} \in L^{\infty}\left(0, T ; \mathcal{L}\left(\mathcal{Y}^{\prime}\right)\right)$, the operator $\hat{\mathcal{A}}_{n}(t) \equiv \hat{\mathcal{A}}-\mathcal{D}_{1} \mathcal{P}_{n-1}(t)$ is the generator of an evolution operator $U_{n}(t, s)$ satisfying $(3.11)$. Then the solution $\mathcal{P}_{n}(t)$ of $(3.14)$ is given by

$\left\langle\mathcal{P}_{n}(t) \Phi, \Psi\right\rangle_{\mathcal{Y}, \mathcal{Y}^{\prime}}=\int_{t}^{T}\left\langle U_{n}^{*}(\sigma, t)\left[\mathcal{D}_{2}(\sigma)+\left(\mathcal{P}_{n-1} \mathcal{D}_{1} \mathcal{P}_{n-1}\right)(\sigma)\right] U_{n}(\sigma, t) \Phi, \Psi\right\rangle_{\mathcal{Y}, \mathcal{Y}^{\prime}} d \sigma$ for all $\Phi, \Psi \in \mathcal{Y}^{\prime}$. Since $U_{n}^{*}\left\{\mathcal{D}_{2}+\mathcal{P}_{n-1} \mathcal{D}_{1} \mathcal{P}_{n-1}\right\} U_{n} \in L^{\infty}\left(\Delta ; \mathcal{L}\left(\mathcal{Y}^{\prime}, \mathcal{Y}\right)\right)$, we obtain $\mathcal{P}_{n} \in L^{\infty}\left(0, T ; \mathcal{L}\left(\mathcal{Y}^{\prime}, \mathcal{Y}\right)\right)$. Moreover if we can verify that $\left\{\mathcal{P}_{n}\right\}$ is bounded in $L^{\infty}\left(0, T ; \mathcal{L}\left(\mathcal{Y}^{\prime}, \mathcal{Y}\right)\right)$, we can find an operator $\mathcal{P}$ as the uniform limit of $\mathcal{P}_{n}$. To this end, we shall show that the sequence $\left\{\mathcal{P}_{n}\right\}$ is a positive monotone nonincreasing sequence of self adjoint operators, that is,

$$
\left\langle\mathcal{P}_{n}(t) \mathbf{h}, \mathbf{h}\right\rangle_{\mathcal{Y}, \mathcal{Y}^{\prime}} \leq\left\langle\mathcal{P}_{n-1}(t) \mathbf{h}, \mathbf{h}\right\rangle_{\mathcal{Y}, \mathcal{Y}^{\prime}} \text { for all } t \in(0, T), \quad \forall \mathbf{h} \in \mathcal{Y}^{\prime}
$$

Indeed, if we put $\mathcal{Q}_{n}=\mathcal{P}_{n}-\mathcal{P}_{n-1}$, then the operator $\mathcal{Q}_{n}$ satisfies from (3.14) that

$$
\left\{\begin{array}{c}
\frac{d}{d t}\left\langle\mathcal{Q}_{n}(t) \mathbf{h}, \tilde{\mathbf{h}}\right\rangle_{\mathcal{Y}^{\prime}, \mathcal{Y}}+\left\langle\hat{\mathcal{A}}_{n}(t) \mathbf{h}, \mathcal{Q}_{n}(t) \tilde{\mathbf{h}}\right\rangle_{\mathcal{Y}^{\prime}, \mathcal{Y}}+\left\langle\mathcal{Q}_{n}(t) \mathbf{h}, \hat{\mathcal{A}}_{n}(t) \tilde{\mathbf{h}}\right\rangle_{\mathcal{Y}, \mathcal{Y}^{\prime}} \\
-\left\langle\mathcal{D}_{1} \mathcal{Q}_{n-1}(t) \mathbf{h}, \mathcal{Q}_{n-1}(t) \tilde{\mathbf{h}}\right\rangle_{\mathcal{Y}^{\prime}, \mathcal{Y}}=0, \quad \mathbf{h}, \quad \tilde{\mathbf{h}} \in \mathcal{Y}, \quad \mathcal{Q}_{n}(T)=0
\end{array}\right.
$$

and the solution of this equation is given by

$$
\left\langle\mathcal{Q}_{n}(t) \mathbf{h}, \tilde{\mathbf{h}}\right\rangle_{\mathcal{Y}, \mathcal{Y}^{\prime}}=-\int_{t}^{T}\left\langle\mathcal{Q}_{n-1} \mathcal{D}_{1} \mathcal{Q}_{n-1}(\sigma) U_{n}(\sigma, t) \mathbf{h}, U_{n}(\sigma, t) \tilde{\mathbf{h}}\right\rangle_{\mathcal{Y}, \mathcal{Y}^{\prime}} d \sigma
$$

for all $\mathbf{h}, \tilde{\mathbf{h}} \in \mathcal{Y}^{\prime}$ by extension, where $\hat{\mathcal{A}}_{n}(t) \equiv \hat{\mathcal{A}}-\mathcal{D}_{1} \mathcal{P}_{n-1}(t)$. Since

$$
\left(R^{-1}(t) v, v\right)_{U}=\left(v, R^{-1}(t) v\right)_{U} \geq \rho\|v\|_{U}
$$

for some $\rho>0$ and for all $v \in U$, we have

$$
\begin{aligned}
\left\langle\mathcal{D}_{1}(t) \mathbf{h}, \mathbf{h}\right\rangle_{\mathcal{Y}^{\prime}, \mathcal{Y}} & =\left\langle\mathcal{B} R^{-1} \Lambda_{U} \mathcal{B}^{*}(t) \mathbf{h}, \mathbf{h}\right\rangle_{\mathcal{Y}^{\prime}, \mathcal{Y}} \\
& =\left(\mathcal{B}^{*}(t) \mathbf{h}, R^{-1} \mathcal{B}^{*}(t) \mathbf{h}\right)_{U} \geq \rho\left\|\mathcal{B}^{*}(t) \mathbf{h}\right\|_{U}^{2}, \quad \forall \mathbf{h} \in \mathcal{Y} .
\end{aligned}
$$

Hence $\mathcal{D}_{1}(t)$ is a nonnegative operator. From the symmetricity of $\mathcal{Q}_{n}$, we have $\left\langle\mathcal{Q}_{n}(t) \mathbf{h}, \mathbf{h}\right\rangle_{\mathcal{Y}, \mathcal{Y}^{\prime}} \leq 0$ for all $\mathbf{h} \in \mathcal{Y}^{\prime}$. This proves (3.16). By induction (3.14), it is easily verified that

$$
0 \leq\left\langle\mathcal{P}_{1}(t) \mathbf{h}, \mathbf{h}\right\rangle_{\mathcal{Y}, \mathcal{Y}^{\prime}} \leq C_{1}\|\mathbf{h}\|_{\mathcal{Y}^{\prime}}^{2}
$$


for some $C_{1}>0$ and all $t \in[0, T]$ and $\mathbf{h} \in \mathcal{Y}^{\prime}$. Therefore, we have from two inequalities (3.16) and (3.17) that

$$
\sup _{t \in[0, T]}\left\langle\mathcal{P}_{n}(t) \mathbf{h}, \mathbf{h}\right\rangle_{\mathcal{Y}, \mathcal{Y}^{\prime}} \leq C_{1}\|\mathbf{h}\|_{\mathcal{Y}^{\prime}}^{2} \text { for all } n \geq 1
$$

which implies that there is a positive self operator $\mathcal{P} \in L^{\infty}\left(0, T ; \mathcal{L}\left(\mathcal{Y}^{\prime}, \mathcal{Y}\right)\right)$ such that $\mathcal{P}_{n}(t)$ converges to $\mathcal{P}(t)$ uniformly in $t$ on $[0, T]$ in the strong operator topology.

Now we note that the evolution operators $U_{n}$ corresponding to the generator $\hat{\mathcal{A}}_{n}(t)$ is the solution of the integral equation

$$
U_{n}(r, t) \mathbf{h}=\hat{S}(r-t) \mathbf{h}-\int_{t}^{r} \hat{S}(r-\sigma)\left(\mathcal{D}_{1} \mathcal{P}_{n-1}\right)(\sigma) d \sigma \mathbf{h} \text { for } \mathbf{h} \in \mathcal{Y}^{\prime} .
$$

Since $\mathcal{D}_{1} \mathcal{P}_{n-1}$ is bounded in $L^{\infty}\left(0, T ; \mathcal{L}\left(\mathcal{Y}^{\prime}\right)\right)$, it follows from Gronwall's lemma that there exists a $C_{2}>0$ such that

$$
\sup _{0<t \leq r<T}\left\|U_{n}(r, t) \mathbf{h}\right\|_{\mathcal{Y}^{\prime}}<C_{2}\|\mathbf{h}\|_{\mathcal{Y}^{\prime}} \text { for all } n .
$$

Let $U$ be the solution of the integral equation

$$
U(r, t) \mathbf{h}=\hat{S}(r-t) \mathbf{h}-\int_{t}^{r} \hat{S}(r-\sigma)\left(\mathcal{D}_{1} \mathcal{P}\right)(\sigma) d \sigma \mathbf{h}
$$

corresponding to the generator $\hat{\mathcal{A}}(t)=\hat{\mathcal{A}}-\mathcal{D}_{1} \mathcal{P}(t)$. By virtue of the Lebesgue dominated convergence theorem, we obtain

$$
U_{n}(r, t) \rightarrow U(r, t) \text { uniformly on } 0<t \leq r<T
$$

in the strong operator topology. Therefore, passing the limit to (3.15) with $\Phi=\mathbf{h}, \Psi=\tilde{\mathbf{h}}$ and $U_{n}(r, t) \rightarrow U(r, t)$ strongly and uniformly in $t$, we obtain

$$
\langle\mathcal{P}(t) \mathbf{h}, \tilde{\mathbf{h}}\rangle_{\mathcal{Y}, \mathcal{Y}^{\prime}}=\int_{t}^{T}\left\langle U^{*}(\sigma, t)\left[\mathcal{D}_{2}(\sigma)+\left(\mathcal{P} \mathcal{D}_{1} \mathcal{P}\right)(\sigma)\right] U(\sigma, t) \mathbf{h}, \tilde{\mathbf{h}}\right\rangle_{\mathcal{Y}, \mathcal{Y}^{\prime}} d \sigma
$$

for all $\mathbf{h}, \tilde{\mathbf{h}} \in \mathcal{Y}^{\prime}$. Recall that $\hat{\mathcal{A}}_{n}(t)$ is the generator of $U_{n}$ satisfying

$$
\left\langle\Phi, U_{n}(r, t) \Psi-\Psi\right\rangle_{\mathcal{Y}, \mathcal{Y}^{\prime}}=\int_{t}^{r}\left\langle\Phi, U_{n}(r, \sigma) \hat{\mathcal{A}}_{n}(\sigma) \Psi\right\rangle_{\mathcal{Y}, \mathcal{Y}^{\prime}} d \sigma \quad \text { for } \Phi \in \mathcal{Y}, \Psi \in \mathcal{H}
$$

Since $\mathcal{D}_{1} \mathcal{P}_{n}(t) \rightarrow \mathcal{D}_{1} \mathcal{P}(t)$ in the strong operator topology for almost every $t$ in $[0, T]$ and $U_{n}(r, t) \rightarrow U(r, t)$ uniformly on $\Delta$ in the strong operator topology, if we take the limit in (3.19), then we have

$$
\langle\Phi, U(r, t) \Psi-\Psi\rangle_{\mathcal{Y}, \mathcal{Y}^{\prime}}=\int_{t}^{r}\langle\Phi, U(r, \sigma) \hat{\mathcal{A}}(\sigma) \Psi\rangle_{\mathcal{Y}, \mathcal{Y}^{\prime}} d \sigma \text { for } \Phi \in \mathcal{Y}, \Psi \in \mathcal{H}
$$

Differentiating (3.20) in $t$ (in real sense) we have

$$
\frac{\partial}{\partial t}\langle\Phi, U(r, t) \Psi\rangle_{\mathcal{Y}, \mathcal{Y}^{\prime}}=-\langle\Phi, U(r, t) \hat{\mathcal{A}}(t) \Psi\rangle_{\mathcal{Y}, \mathcal{Y}^{\prime}} \quad \text { for } \quad \Phi \in \mathcal{Y}, \Psi \in \mathcal{H}
$$


Since the integrand of (3.18) is bounded in $\mathbf{R}, t \rightarrow\langle\mathcal{P}(t) \mathbf{h}, \tilde{\mathbf{h}}\rangle_{\mathcal{Y}, \mathcal{Y}^{\prime}}$ is absolutely continuous and so, it is differentiable almost everywhere in $[0, T]$. As a result, if we differentiate (3.18), then from (3.20) and (3.21) we can verify the following

$$
\begin{aligned}
& \frac{d}{d t}\langle\mathcal{P}(t) \mathbf{h}, \tilde{\mathbf{h}}\rangle_{\mathcal{Y}, \mathcal{Y}^{\prime}}=-\left\langle\left(\mathcal{D}_{2}(t)+\mathcal{P} \mathcal{D}_{1} \mathcal{P}(t)\right) \mathbf{h}, \tilde{\mathbf{h}}\right\rangle_{\mathcal{Y}, \mathcal{Y}^{\prime}} \\
& -\left\langle\mathcal{A}^{*} \mathcal{P}(t) \mathbf{h}, \tilde{\mathbf{h}}\right\rangle_{\mathcal{Y}, \mathcal{Y}^{\prime}}-\left\langle\mathbf{h}, \mathcal{A}^{*} \mathcal{P}(t) \tilde{\mathbf{h}}\right\rangle_{\mathcal{Y}^{\prime}, \mathcal{Y}} \text { for } \mathbf{h}, \tilde{\mathbf{h}} \in \mathcal{H} \quad \text { a.e. } t \in(0, T) .
\end{aligned}
$$

Thus the operator valued function $\mathcal{P}$ satisfies the differential equation (3.13), which proves the existence of $\mathcal{P}$. For the uniqueness, let us assume that $\tilde{\mathcal{P}}$ is another solution of (3.13) and let us define $\mathcal{Q}=\mathcal{P}-\tilde{\mathcal{P}}$. Then the operator $\mathcal{Q}$ satisfies the following equation

$$
\begin{aligned}
& \frac{d}{d t}(\mathcal{Q}(t) \mathbf{h}, \tilde{\mathbf{h}})_{\mathcal{H}}+\langle\hat{\mathcal{A}}(t) \mathbf{h}, \mathcal{Q}(t) \tilde{\mathbf{h}}\rangle_{\mathcal{Y}^{\prime}, \mathcal{Y}}+\langle\mathcal{Q}(t) \mathbf{h}, \hat{\mathcal{A}}(t) \tilde{\mathbf{h}}\rangle_{\mathcal{Y}, \mathcal{Y}^{\prime}} \\
& -\left\langle\mathcal{Q} \mathcal{D}_{1} \mathcal{Q}(t) \mathbf{h}, \tilde{\mathbf{h}}\right\rangle_{\mathcal{Y}^{\prime}, \mathcal{Y}}=0, \quad \mathcal{Q}(T)=0, \quad \mathbf{h}, \tilde{\mathbf{h}} \in \mathcal{H} \quad \text { a.e. } t \in(0, T) .
\end{aligned}
$$

The solution of this equation is given by

$$
\langle\mathcal{Q}(t) \mathbf{h}, \tilde{\mathbf{h}}\rangle_{\mathcal{Y}, \mathcal{Y}^{\prime}}=\int_{t}^{T}\left\langle U^{*}(\sigma, t)\left(\mathcal{Q D}_{1} \mathcal{Q}\right)(\sigma) U(\sigma, t) \mathbf{h}, \tilde{\mathbf{h}}\right\rangle_{\mathcal{Y}, \mathcal{Y}^{\prime}} d \sigma, \quad t \in(0, T)
$$

for all $\mathbf{h}, \tilde{\mathbf{h}} \in \mathcal{Y}^{\prime}$. Since $\sup _{0<t \leq \sigma<T}\|U(\sigma, t)\|_{\mathcal{L}\left(\mathcal{Y}^{\prime}\right)}<\delta$ for some $\delta>0$, we have the following estimate

$$
\left|(\mathcal{Q}(t) \mathbf{h}, \mathbf{h})_{\mathcal{H}}\right| \leq \delta^{2}|\mathbf{h}|_{\mathcal{H}}^{2} \int_{t}^{T}\left\|\mathcal{D}_{1}(\sigma)\right\|_{\mathcal{L}\left(\mathcal{Y}^{\prime}\right)}\|\mathcal{Q}(\sigma)\|_{\mathcal{L}\left(\mathcal{Y}^{\prime}\right)}^{2} d \sigma
$$

for all $\mathbf{h} \in \mathcal{Y}^{\prime}$ and $t \in[0, T]$. That is,

$$
\|\mathcal{Q}(t)\|_{\mathcal{L}\left(\mathcal{Y}^{\prime}\right)} \leq \delta^{2} \int_{t}^{T}\left\|\mathcal{D}_{1}(\sigma)\right\|_{\mathcal{L}\left(\mathcal{Y}^{\prime}\right)}\|\mathcal{Q}(\sigma)\|_{\mathcal{L}\left(\mathcal{Y}^{\prime}\right)}^{2} d \sigma \text { for all } t \in[0, T] .
$$

Thus from the lemma in [7, p. 127], we have $\mathcal{Q}(t)=0$. Now let us consider the existence and uniqueness of the equation (3.13). We have already seen that there exists a unique positive self adjoint operator $\mathcal{P} \in L^{\infty}\left(0, T ; \mathcal{L}\left(\mathcal{Y}^{\prime}, \mathcal{Y}\right)\right)$ that solves $(3.13)$ and $\left(\mathcal{P} \mathcal{D}_{1}\right)^{*}=\mathcal{D}_{1} \mathcal{P}$. Since $\hat{\mathcal{A}}-\left(\mathcal{D}_{1} \mathcal{P}\right)(t)$ is the generator of the evolution operator $U,(3.13)$ has a unique mild solution given by

$$
\langle\mathbf{r}(t), \mathbf{h}\rangle_{\mathcal{Y}, \mathcal{Y}^{\prime}}=\int_{t}^{T}\left\langle\mathcal{P}(s) \mathbf{f}(s)+\mathcal{C}^{*}(s) \Lambda_{M} \mathbf{z}_{d}(s), U(s, t) \mathbf{h}\right\rangle_{\mathcal{Y}, \mathcal{Y}^{\prime}} d s, \quad \forall t \in(0, T)
$$

for all $\mathbf{h} \in \mathcal{Y}^{\prime}$. Now it is clear from (3.22) that $\mathbf{r}(t)$ satisfies (3.13). This finishes an outline of the proof.

Let us define the controller $\mathcal{B}(t): \mathcal{U} \rightarrow \mathcal{V}_{2}^{\prime}$ as

$$
\mathcal{B}(t) v=(0, B(t) v) \text { a.e. } t \in[0, T],
$$

where $B \in L^{\infty}\left(0, T ; \mathcal{L}\left(\mathcal{U}, V_{2}^{\prime}\right)\right)$. Then it is clear from the obvious inclusion that $\mathcal{B}(t) \in \mathcal{L}\left(\mathcal{U}, \mathcal{V}_{2}^{\prime}\right) \subset \mathcal{L}\left(\mathcal{U}, \mathcal{Y}^{\prime}\right)$, and so $\mathcal{B} \in L^{\infty}\left(0, T ; \mathcal{L}\left(\mathcal{U}, \mathcal{Y}^{\prime}\right)\right)$, because of 
$\mathcal{V}_{2}^{\prime} \hookrightarrow \mathcal{V}^{\prime}$. Hence the operator $\mathcal{D}_{1}$ is decomposed by the form of

$$
\mathcal{D}_{1}(t)=\left(\begin{array}{cc}
0 & 0 \\
0 & \mathcal{B} R^{-1} \mathcal{B}^{*}(t)
\end{array}\right)=\left(\begin{array}{cc}
0 & 0 \\
0 & D_{1}(t)
\end{array}\right) \quad \text { a.e. } t \in[0, T] .
$$

Theorem 3.5. Let the controller $\mathcal{B}$ be the one defined by (3.23). Then the Riccati equation can be written by the following four equations:

$P_{0}^{\prime}(t)-P_{2}(t)-P_{1}(t) A_{1}-P_{1}(t) D_{1}(t) P_{2}(t)=D_{21}(t), t \in(0, T)$,

$P_{1}^{\prime}(t)-P_{3}(t)+P_{0}(t)-P_{1}(t) A_{2}-P_{1}(t) D_{1}(t) P_{3}(t)=D_{22}(t), t \in(0, T)$,

$P_{2}^{\prime}(t)+A_{1} P_{0}(t)-A_{2} P_{2}(t)-P_{3}(t) A_{1}-P_{3}(t) D_{1}(t) P_{2}(t)=0, t \in(0, T)$,

$P_{3}^{\prime}(t)+A_{1} P_{1}(t)-A_{2} P_{3}(t)+P_{2}(t)-P_{3}(t) A_{2}-P_{3}(t) D_{1}(t) P_{3}(t)=0, t \in(0, T)$,

with

$$
P_{1}(T)=P_{2}(T)=P_{3}(T)=P_{4}(T)=0,
$$

where

$$
\mathcal{P}(t)=\left(\begin{array}{ll}
P_{0}(t) & P_{1}(t) \\
P_{2}(t) & P_{3}(t)
\end{array}\right) \quad \text { and } \quad \mathcal{D}_{2}(t)=\left(\begin{array}{cc}
D_{21}(t) & D_{22}(t) \\
0 & 0
\end{array}\right), \quad t \in[0, T] .
$$

Proof. From (3.13), it follows that $\mathcal{P}$ satisfies

$$
\frac{d}{d t} \mathcal{P}+\mathcal{P} \mathcal{A}+\mathcal{A}^{*} \mathcal{P}-\mathcal{P} \mathcal{D}_{1} \mathcal{P}+\mathcal{D}_{2}=0 \quad \text { in }(0, T), \quad \mathcal{P}(T)=0
$$

The componentwise differential equalities for $P_{i}(t), i=0,1,2,3$ follow easily followed from recalling that

$$
\mathcal{A}=\left(\begin{array}{cc}
0 & I \\
-A_{1} & -A_{2}
\end{array}\right), \quad \mathcal{A} *=\left(\begin{array}{cc}
0 & -I \\
A_{1} & -A_{2}
\end{array}\right)
$$

and substituting these operators $\mathcal{A}, \mathcal{A}^{*}$ into (3.24).

\section{References}

[1] N. U. Ahmed and K. L. Teo, Optimal Control of Distributed Parameter Systems, North Holland, 1981.

[2] J. M. Ball, Strongly continuous semigroups, weak solutions, and the variation of constants formula, Proc. Amer. Math. Soc. 63 (1977), no. 2, 370-373.

[3] H. T. Banks, K. Ito, and Y. Wang, Well Posedness for damped second order systems with unbounded input operators, Differential Integral Equations 8 (1995), no. 3, 587-606.

[4] H. T. Banks, F. Kappel, and Y. Wang, Weak solutions and differentiability for size structured population models, Estimation and control of distributed parameter systems (Vorau, 1990), 35-50, Internat. Ser. Numer. Math., 100, Birkhäuser, Basel, 1991.

[5] V. Barbu, Extended algebraic Riccati equations in the abstract hyperbolic case, Nonlinear Anal. 40 (2000), no. 1-8, Ser. A: Theory Methods, 105-129.

[6] F. Bucci, A Dirichlet boundary control problem for the strongly damped wave equation, SIAM J. Control Optim. 30 (1992), no. 5, 1092-1100.

[7] R. W. Carroll, Abstract Methods in Partial Differential Equstions, Harper and Row, New York, 1969.

[8] R. F. Curtain and A. J. Pritchard, Infinite Dimensional Linear Systems Theory, Lecture Notes in Control and Information Sciences, Springer-Verlag, Vol. 8, 1978. 
[9] R. F. Curtain and Salamon, Finite dimensional compensators for infinite dimensional systems with unbounded input operators, SIAM J. Control Optim. 24 (1986), no. 4, 797-816.

[10] R. Dautray and J. L. Lions, Mathematical Analysis and Numerical Methods for Science and Technology, Springer-Verlag, Vol. 5, Evolution Problems, 1992.

[11] F. Flandoli, Riccati equation arising in a boundary control problem with distributed parameters, SIAM J. Control Optim. 22 (1984), no. 1, 76-86.

[12] J. Ha, Optimal control problems for hyperbolic distributed parameter systems with damping terms, Doctor Thesis, The Graduate School of Science and Technology, Kobe University, 1996, August.

[13] J. Ha and S. Nakagiri, Existence and regularity of weak solutions for semilinear second order evolution equations, Funkcial. Ekvac. 41 (1998), no. 1, 1-24.

[14] J. Ha, S. Nakagiri, and H. Tanabe, Fréchet differentiability of solution mappings for semilinear second order evolution equations, J. Math. Anal. Appl. 346 (2008), no. 2, 374-383.

[15] A. Haraux, Linear semigroups in Banach spaces, Semigroups, theory and applications, Vol. II (Trieste, 1984), 93-135, Pitman Res. Notes Math. Ser., 152, Longman Sci. Tech., Harlow, 1986.

[16] R. E. Kalman, Contributions to the theory of optimal control, Bol. Soc. Mat. Mexicana 5 (1960), 102-119.

[17] I. Lasiecka and R. Triggiani, Differential and Algebraic Riccati Equations with Application to Boundary/Point Control Problems: Continuous Theory and Approximation Theory, Lecture Notes in Control and Information Sciences, Springer-Verlag, Vol. 164, 1991.

[18] - Dirichlet boundary control problem for parabolic equations with quadratic cost: Analyticity and Riccati's feedback synthesis, SIAM J. Control Optim. 21 (1983), no. 1, $41-67$.

[19] Control theory for partial differential equations: continuous and approximation theories. II. Abstract hyperbolic-like systems over a finite time horizon, Encyclopedia of Mathematics and its Applications, Vol. 75. Cambridge University Press, Cambridge, 2000.

[20] J. L. Lions, Optimal Control of Systems Governed by Partial Differential Equations, Springer-Verlag Berlin Heidelberg New York, 1971.

[21] J. L. Lions and E. Magenes, Non-Homogeneous Boundary Value Problems and Applications I, II, Springer-Verlag Berlin Heidelberg New York, 1972.

[22] A. Pazy, Semigroups of Linear Operators and Applications to Partial Differential Equations, Springer-Verlag, 1983.

[23] A. J. Pritchard and D. Salamon, The linear quadratic control problem for infinite dimensional systems with unbounded input and output operators, SIAM J. Control Optim. 25 (1987), no. 1, 121-144.

[24] H. Tanabe, Equations of Evolution, Pitman, London, 1979.

[25] R. Triggiani, Optimal quadratic boundary control problem for wave- and wave-like equations with high internal damping: An abstract approach, Lecture Notes in Pure and Appl. Math., 165, Control of partial differential equations (Trento, 1993), 215-270, Dekker, New York, 1994.

JUNHONG HA

School of Liberal ARTS

Korea University of Technology and Education

Cheonan 330-708, Korea

E-mail address: hjh@koreatech.ac.kr 
SHIN-ICHI NAKAGIRI

Department of Applied Mathematics Faculty of Engineering

KoBE UNIVERSITY

KOBE 657-8501, JAPAN

E-mail address: nakagiri@cs.kobe-u.ac.jp 